\title{
Wearable-based pedestrian inertial navigation with constraints based on biomechanical models
}

\author{
Dina Bousdar Ahmed* and Kai Metzger ${ }^{\dagger}$ \\ *Institute of Communications and Navigation \\ German Aerospace Center (DLR), Munich, Germany \\ Email: dina.bousdarahmed@dlr.de \\ $\dagger$ Technical University of Munich, Germany \\ Email: kai_metzger@web.de
}

\begin{abstract}
Our aim in this paper is to analyze inertial navigation systems (INSs) from the biomechanical point of view. We wanted to improve the performance of a thigh INS by applying biomechanical constraints. To that end, we propose a biomechanical model of the leg. The latter establishes a relationship between the orientation of the thigh INS and the kinematic motion of the leg. This relationship allows to observe the effect that the orientation errors have in the expected motion of the leg. We observe that the errors in the orientation estimation of an INS translate into incoherent human motion. Based on this analysis, we proposed a modified thigh INS to integrate biomechanical constraints. The results show that the proposed system outperforms the thigh INS in $\mathbf{5 0 \%}$ regarding distance error and $32 \%$ regarding orientation error. Our approach allows us to improve the performance of INS using solely inertial measurements and biomechanical constraints. Additionally, it allows us to state that the performance of an INS can be improved by correcting the incoherent orientations.
\end{abstract}

\section{INTRODUCTION}

The protection of vulnerable road users (VRUs), i.e. pedestrians and cyclists, is a topic that is gaining attention. In 2015, $15.7 \%$ of the car accidents in Germany resulted in damages to VRUs [1]. Had the pedestrian's position been known to the car drivers, lives could have been saved.

To protect VRUs, it is crucial to know their location, which can be done using wearable devices. The latter are equipped, not only with a variety of sensors, but have processing capabilities that are expected to be similar to smart phones and computers [2]. Wearable devices, or simply wearables, can locate a pedestrian in GPS-denied environments, e.g. urban canyons.

Among the technologies integrated in wearables, we focus on using inertial sensors to locate a pedestrian. The most important challenge of inertial navigation is the drift of the heading. The drift is the error in the heading which is caused, mainly, by the bias in the gyroscopes [3].

There is a number of different techniques to reduce the effect of the drift in the position estimation of an INS. Some examples are sensor fusion, landmarks detection or the combination of multiple inertial measurement units (IMUs).

Sensor fusion in inertial navigation combines inertial sensors with a different technology. For example, inertial positioning can be combined with a light detection and ranging sensor to correct the heading estimation [4]. The combination of inertial sensors and WiFi measurements is useful in indoor environments. Chen et al. use the position estimated through WiFi measurements to correct the position estimates of an inertial navigation system [5]. The latter is based on a smartphone.

The detection of known landmarks, e.g. turns, elevators, etc, can improve the position estimation. Chen et al. [5] incorporate landmarks to improve the performance of their navigation system. Munoz et al. [6] use also landmarks to correct directly the heading estimation of a thigh-mounted inertial navigation system.

The combination of multiple IMUs has been explored by Skog et al., among others. The authors develop a sensor array to estimate more accurate acceleration and turn rate than a single-IMU sensor [7]. A different approach is followed in [8], where the authors combine the output of two inertial navigation systems. The latter are based on two foot-mounted IMUs, one on each foot respectively. A constraint is set for the maximum allowed separation between the two position estimates. This approach is useful only when the heading of each INS drifts in opposite directions.

An interesting approach is followed by Ahmadi et al. [9]. They use a biomechanical model of the leg to aid gait reconstruction using inertial sensors. Such approach could be extended to other applications, for example, inertial navigation.

Nobody, until now, has applied biomechanical models to inertial navigation. In fact, the relationship between human biomechanics and the orientation errors is unknown. The understanding of this relationship could be used to improve the performance of inertial navigation systems.

The goal of this paper is to analyze whether information about human biomechanical motion can improve the position estimation of an INS. We use two IMUs attached to the pedestrian's upper thigh and foot, respectively.

\section{METHODS}

This section describes the steps followed to understand how biomechanical knowledge can improve the performance of an INS. Our work is organized in three steps.

Firstly, we provide a biomechanical model of the human leg. Secondly, we analyze the orientation of the thigh-mounted INS 


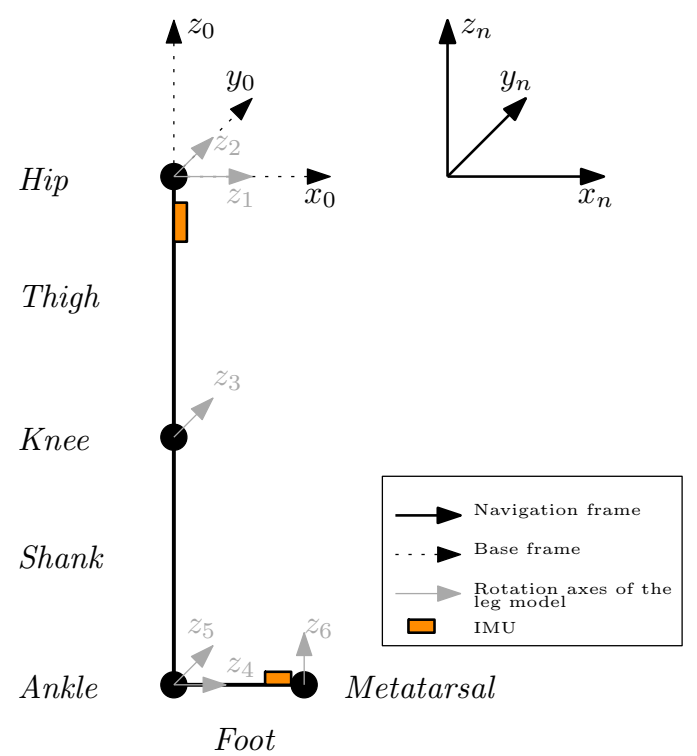

Fig. 1: Leg model consisting of joints (circles) and links (solid lines).

through the leg model. Finally, we propose modifications to a thigh INS based on the previous analysis.

\section{A. Biomechanical 5-DoF-model}

In order to understand the biomechanics of the human leg, a leg model is proposed. The model represents the human leg as a set of joints and links, see Figure 1. The joints are chosen to be the hip, knee, ankle and metatarsal. The links, which connect two consecutive joints, are the thigh, shank and foot.

The model allows each joint to rotate around one or more rotational axes. Each rotational axis is a degree-of-freedom (DoF). In this case, we have chosen a model with five DoF: two for the hip $\left(z_{1}, z_{2}\right)$, one for the knee $\left(z_{3}\right)$ and two for the ankle $\left(z_{4}, z_{5}\right)$, see Figure 1 . The axis $z_{6}$ is introduced only to estimate the position of the metatarsal. This axis is not a degree of freedom in the current implementation.

The model is used to estimate the structure of the leg, i.e. the position of each joint. Each joint position is represented in the base frame, see Figure 1. The base frame, indicated by the triplet $\left\{x_{0}, y_{0}, z_{0}\right\}$, is a fixed frame of free choice. In our case, it was defined to be aligned with the navigation frame, see Figure 1, which is also fixed.

In order to describe the structure of the leg, the DenavitHartenberg parameters are used [10]. These parameters are used in robotics to estimate the position of the joints of robotic structures. In our case, the robotic structure is the leg model.

Given two rotational axes $z_{i-1}$ and $z_{i}$, the DenavitHartenberg parameters that represent their relative position are:

1) $d_{i}:$ link offset distance,

2) $\theta_{i}$ : link rotation angle,

3) $a_{i}$ : link length,

4) $\alpha_{i}$ : link twist angle.
These parameters are defined for each rotational axis of the structure. The Denavit-Hartenberg parameters of the leg model in Figure 1 are given in Table I. The angles $\theta_{i}\{i=1, \ldots, 5\}$ are defined to coincide with either the roll or pitch of their associated joint.

TABLE I: Denavit-Hartenberg parameters of the leg model in Figure 1. Each angle $\theta_{i}$, in degrees, is the rotation around its axis. The lengths of the thigh, shank and foot are given by $l_{\mathrm{t}}$, $l_{\mathrm{s}}$ and $l_{\mathrm{f}}$ respectively.

\begin{tabular}{ccccc}
\hline Link no. & $\boldsymbol{d}_{\boldsymbol{i}}$ & $\boldsymbol{\theta}_{\boldsymbol{i}}$ & $\boldsymbol{a}_{\boldsymbol{i}}$ & $\boldsymbol{\alpha}_{\boldsymbol{i}}$ \\
\hline 1 & 0 & $-90^{\circ}$ & 0 & $-90^{\circ}$ \\
2 & 0 & $90^{\circ}+\theta_{1}$ & 0 & $-90^{\circ}$ \\
3 & 0 & $\theta_{2}$ & $l_{\mathrm{t}}$ & $0^{\circ}$ \\
4 & 0 & $180^{\circ}+\theta_{3}$ & $l_{\mathrm{s}}$ & $-90^{\circ}$ \\
5 & 0 & $180^{\circ}+\theta_{4}$ & 0 & $-90^{\circ}$ \\
6 & 0 & $90^{\circ}+\theta_{5}$ & $l_{\mathrm{f}}$ & $-90^{\circ}$ \\
\hline
\end{tabular}

Given the rotation angles $\theta_{i},\{i=1, \ldots, 5\}$, the leg model estimates the position of the joints. For that purpose, the DenavitHartenberg parameters are used to compute the homogenous matrix $H_{i}^{i-1}$. The latter is a $4 \times 4$ matrix used in geometry to represent the relative position of the two reference frames $i-1$ and $i$ :

$$
H_{i}^{i-1}=\left[\begin{array}{cccc}
\cos \theta_{i} & -\sin \theta_{i} \cdot \cos \alpha_{i} & \sin \theta_{i} \cdot \sin \alpha_{i} & a_{i} \cdot \cos \theta_{i} \\
\sin \theta_{i} & \cos \theta_{i} \cdot \cos \alpha_{i} & -\cos \theta_{i} \cdot \sin \alpha_{i} & a_{i} \cdot \sin \theta_{i} \\
0 & \sin \alpha_{i} & \cos \alpha_{i} & d_{i} \\
0 & 0 & 0 & 1
\end{array}\right] .
$$

The kinematic chain is the successive multiplication of homogenous matrices to compute the position of the desired joint or joints. In this case, we want to estimate the position of the metatarsal $\left(z_{6}\right)$ in the base frame, see Figure 1. For that purpose, the matrix $H_{6}^{0}$ is computed as:

$$
H_{6}^{0}=H_{1}^{0} \cdot H_{2}^{1} \cdot \ldots \cdot H_{6}^{5} .
$$

The position of the metatarsal is given by the first three elements of the last column of $H_{6}^{0}$.

An example of use of the leg model is shown in Figure 2. In the latter, the values of the angles $\theta_{1}$ and $\theta_{2}$ have been taken from [11]. The blue crosses represent all possible positions of the metatarsal when the hip rotates $\theta_{1}$ and $\theta_{2}$ while maintaining the leg stretched.

Until now, we have presented a leg model to represent human motion. The next step is to understand human motion, which is known from medicine studies [11].

There is a range of motions that a person can perform naturally. These motions are defined as coherent, e.g. bending the knee $90^{\circ}$. In contrast, the physical limitations of the human body define the incoherent motions. An example of incoherent motion is rotating the foot $180^{\circ}$.

It is possible to analyze, by using the aforementioned leg model, whether a set of rotation angles $\theta_{i},\{i=1, \ldots, 5\}$, are coherent or incoherent. For that purpose, the set of rotation angles are used to estimate the position of the joints. Then, these positions are compared to the expected ones that comprise the coherent motions. 


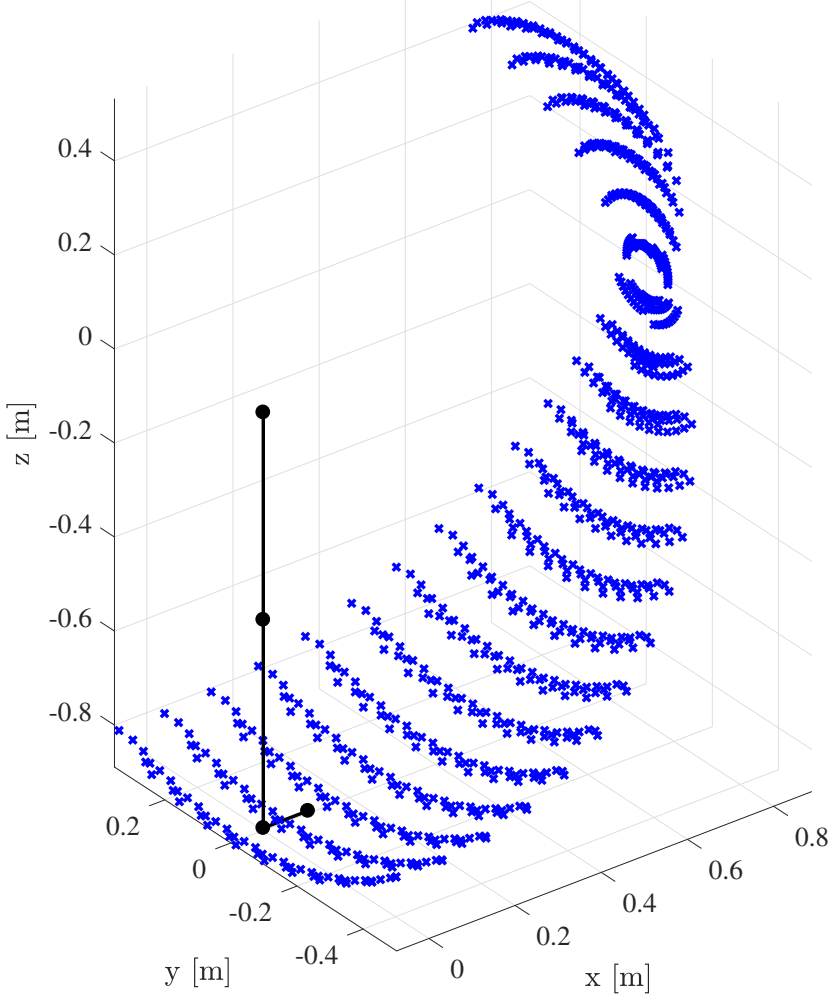

Fig. 2: Possible positions of the metatarsal (blue crosses) when $\theta_{1}=\left[-30^{\circ}, 20^{\circ}\right]$ and $\theta_{2}=\left[-120^{\circ}, 10^{\circ}\right]$. The remaining degrees of freedom are set to $0^{\circ}$.

The next step of our work, outlined in the section below, is to extend the analysis to the angles estimated by an inertial navigation system.

\section{B. Relationship of inertial orientation to leg biomechanics}

The model proposed in the previous section is now used to analyze the orientation estimation of an INS. In our case, two IMUs are available: one is placed on the upper thigh and another one on the front part of the foot, see Figure 1. The INS uses a thigh-mounted IMU, see [12] and references therein.

The thigh INS estimates, along with the 2D-position of the pedestrian, the orientation of the thigh. The orientation is represented by the Euler angles, namely the roll $(\phi)$, the pitch $(\theta)$ and the yaw $(\phi)$.

The axes $z_{1}$ and $z_{2}$ in Figure 1 are defined to coincide with the $\mathrm{x}$-axis and $\mathrm{y}$-axis, respectively, of the thigh INS. Therefore, the angles $\theta_{1}$ and $\theta_{2}$ are equivalent to the roll $(\phi)$ and pitch $(\theta)$ estimated by the thigh INS, i.e. $\theta_{1}=\phi$ and $\theta_{2}=\theta$.

In order to analyze the coherence of $\phi$ and $\theta$, all angles $\theta_{i}$, $\{i=1, \ldots, 5\}$, are required. As previously mentioned, $\theta_{1}$ and $\theta_{2}$ equal to $\phi$ and $\theta$ respectively. Only $\theta_{i},\{i=3,4,5\}$, are missing. The foot-IMU is used to estimate them.

The stance phase of the foot can be detected with the foot IMU. The stance phase is the period of time during which the foot is contact with the ground. Upon detection of the stance

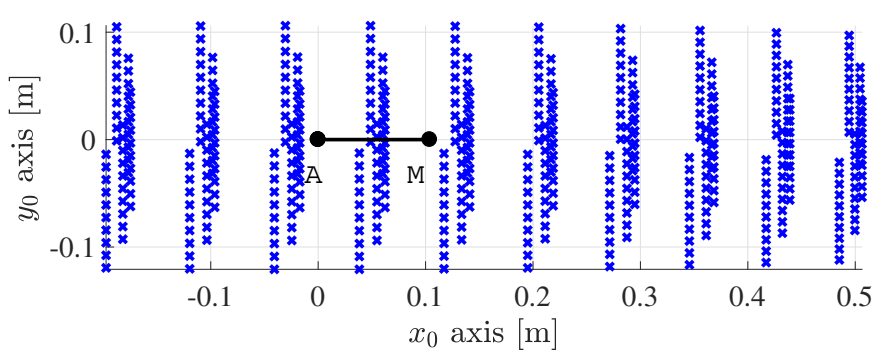

Fig. 3: Comfort zone of the metatarsal during the stance phase. The cross marks are computed with the orientation of the hip during the stance phase. They represent the coherent positions of the metatarsal. The limits of the comfort zone are $\phi=$ $\left[-3.5^{\circ}, 3.5^{\circ}\right]$ and $\theta=\left[-28^{\circ}, 18^{\circ}\right] . A$ stands for ankle and $M$ for metatarsal.

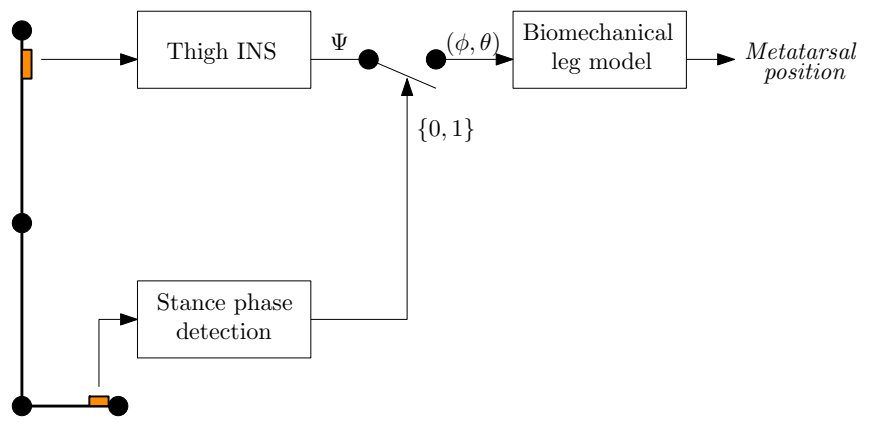

Fig. 4: Estimation of the metatarsal positions according to the orientation of the thigh. $\Psi$ is the orientation vector, i.e. $\Psi=$ $(\phi, \theta, \psi)$.

phase, the angles $\theta_{3}, \theta_{4}$ and $\theta_{5}$ can be approximated by $0^{\circ}$ each.

The set of coherent metatarsal positions during the stance phase is defined as comfort zone. The latter is presented in Figure 3. The limits of the comfort zone, which are given by the limits of the hip during the stance phase, are adapted from [13].

The analysis is summarized in Figure 4. When the stance phase is detected, see Figure 4, the roll and pitch of the thigh INS are input to the leg model. The latter estimates the position of the metatarsal according to the thigh's orientation.

If the roll $(\phi)$ and pitch $(\theta)$ estimates in Figure 4 are coherent, the estimated position of the metatarsal is within the comfort zone. An example is given in Figure 5. Each green-squared mark represents a metatarsal position estimated according to Figure 4. Since the green-squared marks are within the comfort zone, the roll and pitch estimates used to generate them are coherent.

The black-circled marks in Figure 5 are generated according to Figure 4 as well. In this case, the roll and pitch estimates are clearly incoherent because the estimated metatarsal positions are outside the comfort zone.

One of the innovative steps of this work is to relate the orientation estimation of a thigh INS with the biomechanical 


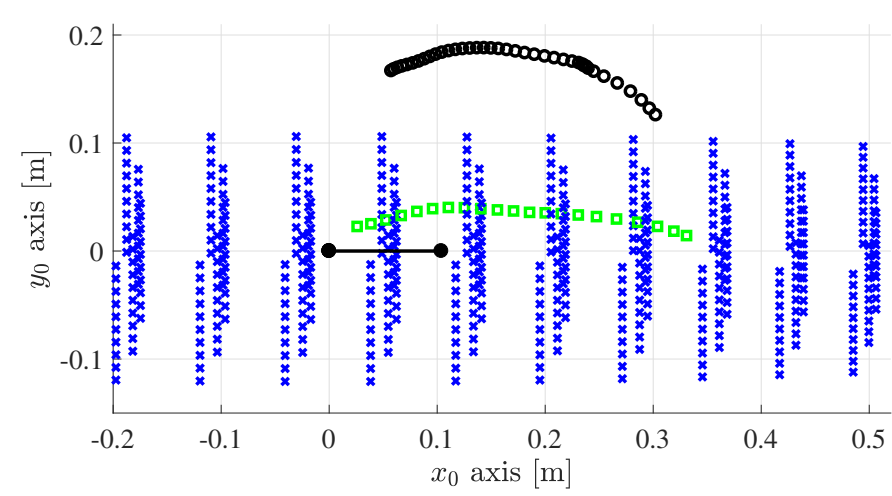

Fig. 5: Comfort zone (blue crosses). The green-squared marks and black-circled marks are position estimates of the metatarsal during two different stance phases. These marks are estimated according to Figure 4.

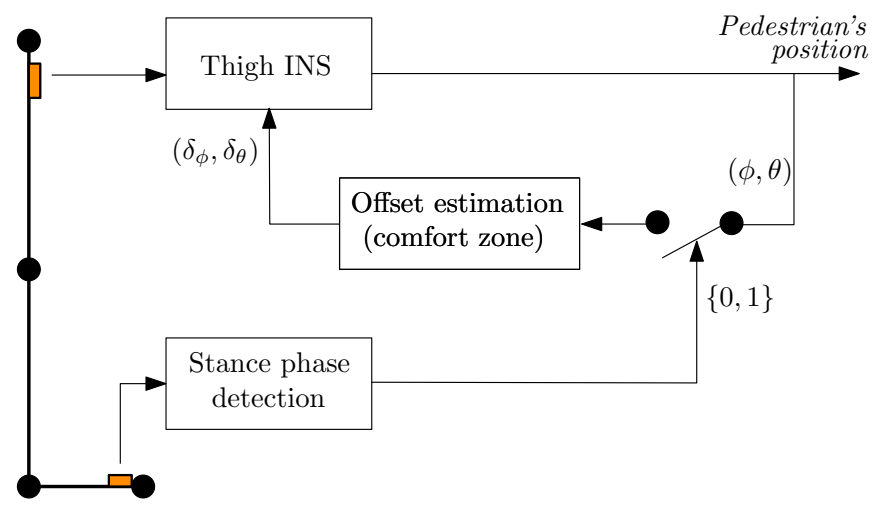

Fig. 6: Inertial navigation system with biomechanical constraints.

behaviour of the leg. This relationship allows for analyzing the orientation from a different point of view: the biomechanical one. As exemplified in Figure 5, the orientation of an INS is coherent if the estimated metatarsal positions are within the limits of the comfort zone.

\section{Model integration in INS}

In this section, we propose an INS that integrates the biomechanical constraints derived from the previous analysis. The proposed system is presented in Figure 6.

Upon detection of the stance phase, see Figure 6, the roll $(\phi)$ and pitch $(\theta)$ estimates of the thigh INS are analysed. The analysis consists of checking whether the maximum and minimum of the roll and pitch of the thigh are within the comfort zone, see Figure 7.

If the roll exceeds the limits of the comfort zone, the roll correction $\left(\delta_{k}^{\phi}\right)$ is estimated as:

$$
\delta_{k}^{\phi}=\left\{\begin{array}{lr}
\phi_{\max }-\max \left(\phi_{\boldsymbol{k}}\right) ; & \text { if } \max \left(\phi_{\boldsymbol{k}}\right)>\phi_{\max }, \\
\phi_{\min }-\min \left(\phi_{\boldsymbol{k}}\right) ; & \text { if } \min \left(\phi_{\boldsymbol{k}}\right)<\phi_{\min }, \\
0 ; & \text { otherwise }
\end{array}\right.
$$

where $\phi_{\max }$ and $\phi_{\min }$ denote the maximum and minimum roll of the comfort zone, respectively. $\phi_{k}$ is the vector of roll

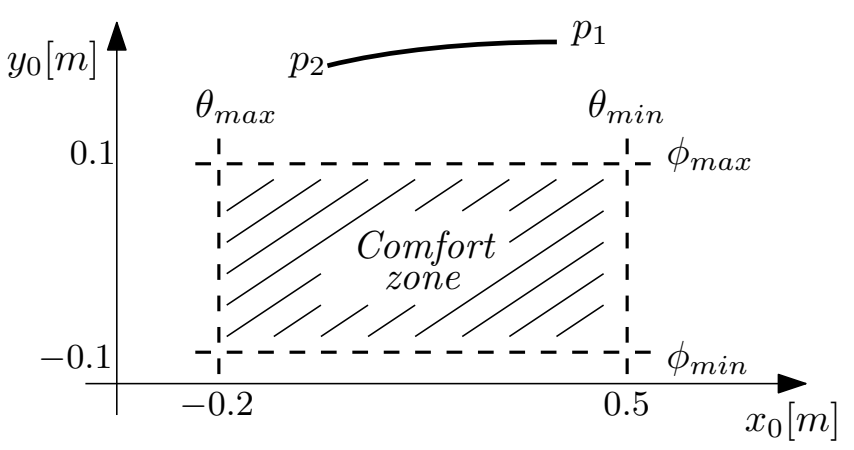

Fig. 7: Limits of the comfort zone. The estimated metatarsal positions, thick-solid line, exceed the comfort zone in the roll. The ends of the metatarsal positions, $p_{1}$ and $p_{2}$, equal to $\boldsymbol{p}_{\mathbf{1}}=\left(\max \left(\phi_{\boldsymbol{k}}\right), \min \left(\boldsymbol{\theta}_{\boldsymbol{k}}\right)\right)$ and $\boldsymbol{p}_{\mathbf{2}}=\left(\min \left(\phi_{\boldsymbol{k}}\right), \max \left(\boldsymbol{\theta}_{\boldsymbol{k}}\right)\right)$.

values during the stance phase at time $k, \max (\cdot)$ denotes the function maximum of the argument and $\min (\cdot)$ denotes the function minimum of the argument.

Similarly, the correction of the pitch $\left(\delta_{k}^{\theta}\right)$ is estimated as follows:

$$
\delta_{k}^{\theta}=\left\{\begin{array}{lr}
\theta_{\max }-\max \left(\boldsymbol{\theta}_{\boldsymbol{k}}\right) ; & \text { if } \max \left(\boldsymbol{\theta}_{\boldsymbol{k}}\right)>\theta_{\max }, \\
\theta_{\min }-\min \left(\boldsymbol{\theta}_{\boldsymbol{k}}\right) ; & \text { if } \min \left(\boldsymbol{\theta}_{\boldsymbol{k}}\right)<\theta_{\min }, \\
0 ; & \text { otherwise, }
\end{array}\right.
$$

where $\theta_{\max }$ and $\theta_{\min }$ are the maximum and minimum pitch of the comfort zone respectively, and $\boldsymbol{\theta}_{\boldsymbol{k}}$ is the vector of pitch values during the stance phase at time $k$.

The corrections $\left(\delta_{k}^{\phi}, \delta_{k}^{\theta}\right)$ are fed back to the thigh INS, see Figure 6. The thigh INS estimates the orientation through an unscented Kalman filter (UKF) [12]. The filter uses $\left(\delta_{k}^{\phi}, \delta_{k}^{\theta}\right)$ to correct the roll and pitch estimates.

The corrections $\left(\delta_{k}^{\phi}, \delta_{k}^{\theta}\right)$ are used as a control vector during the prediction stage of the UKF. To apply the control vector, the correction matrix $\Delta_{k}$ is computed:

$$
\boldsymbol{\Delta}_{\boldsymbol{k}}=\left[\begin{array}{ccc}
\cos \left(\delta_{k}^{\theta}\right) & 0 & \sin \left(\delta_{k}^{\theta}\right) \\
0 & 1 & 0 \\
-\sin \left(\delta_{k}^{\theta}\right) & 0 & \cos \left(\delta_{k}^{\theta}\right)
\end{array}\right] \cdot\left[\begin{array}{ccc}
1 & 0 & 0 \\
0 & \cos \left(\delta_{k}^{\phi}\right) & -\sin \left(\delta_{k}^{\phi}\right) \\
0 & \sin \left(\delta_{k}^{\phi}\right) & \cos \left(\delta_{k}^{\phi}\right)
\end{array}\right] .
$$

The matrix $\Delta_{k}$ corrects the predicted orientation matrix $C_{k}$ :

$$
C_{k}^{\prime}=C_{k} \cdot \Delta_{k},
$$

where $\boldsymbol{C}_{\boldsymbol{k}}$ is computed through the predicted Euler angles $\left(\phi_{k}, \theta_{k}, \psi_{k}\right)$ [14]. The new predicted Euler angles $\left(\phi_{k}^{\prime}, \theta_{k}^{\prime}, \psi_{k}^{\prime}\right)$ can be computed through the corrected orientation matrix $C_{k}^{\prime}$ [14].

It is worth highlight that the corrections in the roll and pitch $\left(\phi_{k}^{\prime}, \theta_{k}^{\prime}\right)$ might lead to an improved heading $\left(\psi_{k}^{\prime}\right)$. The reason is that (6) modifies not only the roll and the pitch, but also the heading.

The estimation of roll and pitch corrections based on human biomechanics is another innovative step of this work. These corrections are intended to make the orientation estimation of an INS coherent with the motion expected from a human while walking. 


\section{Evaluation}

This section describes the methodology to evaluate INSs. The evaluation methodology is applied to the system presented in Section II-C and to the original thigh INS. The objective is to compare both systems to assess the effect of the biomechanical constraints in the position estimation.

\section{A. Evaluation data set}

The methodology to evaluate inertial navigation systems is described in detail in [13]. In the following, a summary is presented for completeness.

The evaluation methodology consists of ground truth points (GTPs), i.e. points whose location is known accurately. These points are visited during the walk. Then, the position estimated by the INS for these points is compared to their true position.

In order to evaluate the navigation systems, two metrics are used: the distance error $\left(e_{\mathrm{d}}\right)$ and the orientation error $\left(e_{\psi}\right)$.

$$
\begin{aligned}
& e_{\mathrm{d}}=d_{i j}^{\mathrm{g}}-d_{i j}^{\mathrm{w}}, \\
& e_{\psi}=\psi_{i j}^{g}-\psi_{i j}^{w},
\end{aligned}
$$

where $d_{i j}^{\mathrm{g}}$ refers to the true distance between the $i$-th and $j$-th GTP. Similarly, $d_{i j}^{\mathrm{w}}$ is the distance estimated by the INS under evaluation between the points $i$ and $j$. The definition of the superindices is the same for (8), where $\psi_{i j}$ denotes the angle between the $i$-th and $j$-th GTP.

The distance error $\left(e_{\mathrm{d}}\right)$ and orientation error $\left(e_{\psi}\right)$ are computed for consecutive GTPs, i.e. $j=i+1$. The reason is that the walks in [13] were designed with approximately straight trajectories between consecutive GTPs. Only in these cases, (7) and (8) represent the distance and orientation error.

The set of walks comprises 17 walks with a total of 149 ground truth points [13].

\section{B. Results and Discussion}

The systems under evaluation are the original thigh INS [12] and the navigation system proposed in Section II-C. Both systems are used to process the set of walks presented in the previous section. Equations (7) and (8) are used to evaluate the processed walks. The results are presented in Table II.

TABLE II: Distance error $\left(e_{\mathrm{d}}\right)$ and orientation error $\left(e_{\psi}\right)$ given as $\mu \pm \sigma$, where $\mu$ is the mean and $\sigma$ the standard deviation.

\begin{tabular}{lcc}
\hline & $\boldsymbol{e}_{\mathrm{d}}[\boldsymbol{m}]$ & $\boldsymbol{e}_{\boldsymbol{\psi}}\left[^{\circ}\right]$ \\
\hline Thigh INS & $0.4 \pm 1.8$ & $14.9 \pm 28.8$ \\
Proposed INS & $0.2 \pm 1.2$ & $10.1 \pm 31.3$ \\
\hline
\end{tabular}

Given the results in Table II, we can state that the proposed INS outperforms the thigh INS. That is, the performance of an INS can be improved if knowledge about biomechanical motion is included in the positioning algorithm.

The biomechanical constraints affect not only the orientation error, but also the distance error. The latter is influenced because the thigh INS uses the pitch angle to compute the step length. Therefore, the correction of the pitch angle also modifies the distance error.

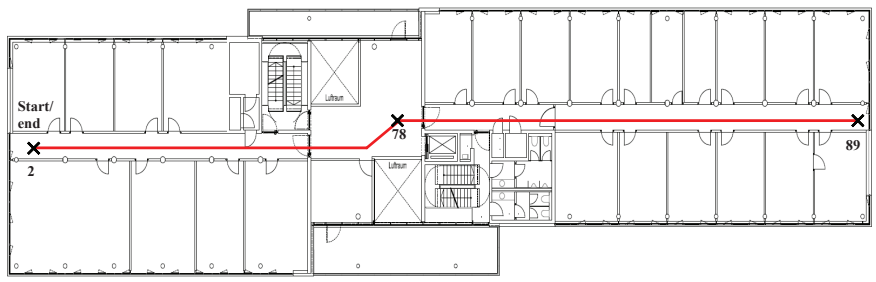

Fig. 8: Floor plan where some of the walks to evaluate the systems were done. The red solid line indicates the approximate trajectory. The crosses are the ground truth points used to evaluate the INSs.

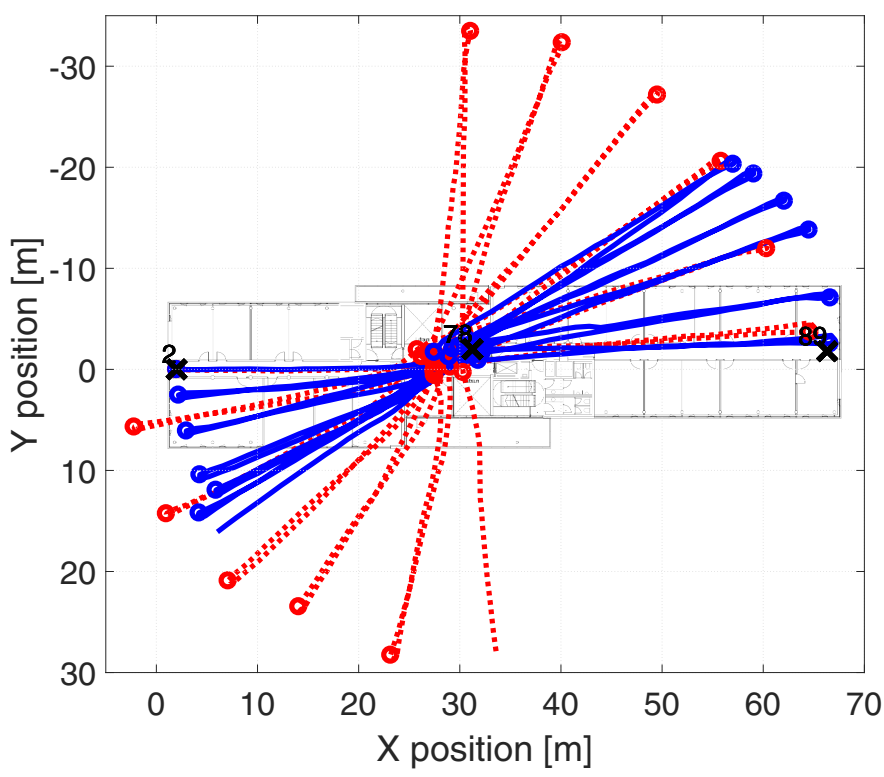

Fig. 9: Odometries estimated by the thigh INS (red-dashed line) and the proposed INS (blue-solid). In this example, the trajectory in Figure 8 was done six times back and forth. The crosses are the GTPs visited during the walk, see Figure 8. The circle marks are the position of the GTPs estimated by each system.

In average, the proposed INS is $50 \%$ better than the thigh INS in distance error. Regarding the average orientation error, the proposed INS is $32 \%$ better than the thigh INS.

Figure 8 is an example of the approximate path of one of the walks. We can observe in Figure 9 that the system with biomechanical constraints leads to a less erroneous odometry than the thigh INS.

It is important to highlight that the biomechanical constraints affect, especially, the orientation error. Figure 10 shows that the orientation error, when the biomechanical constraints are applied, is approximately $60 \%$ of the original error at the end of the walk. In contrast, Figure 11 shows that both systems fluctuate similarly regarding the distance error. The values of the latter are also similar in either INS.

There were some unexpected results during the evaluation of the proposed INS. In some walks, the odometry of the proposed method had larger orientation errors than the original 


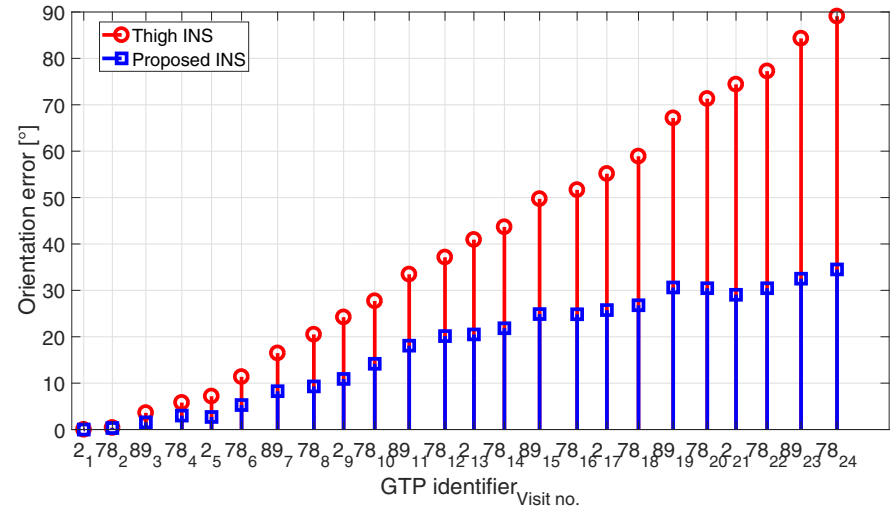

Fig. 10: Orientation error $\left(e_{\psi}\right)$ of the trajectories in Figure 9. The $\mathrm{x}$-axis indicates the GTP identifier, see Figure 8 at which the error is computed. The subindex indicates the order in which the GTP was visited.

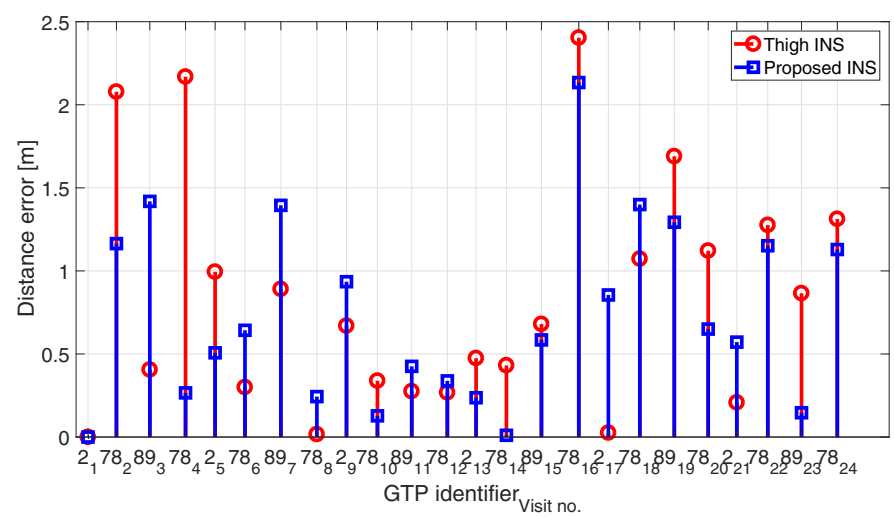

Fig. 11: Distance error $\left(e_{\mathrm{d}}\right)$ of the trajectories in Figure 9. The $\mathrm{x}$-axis indicates the GTP identifier, see Figure 8 at which the error is computed. The subindex indicates the order in which the GTP was visited.

odometry. We think the reason is that the main incoherency lies on the heading $(\psi)$ rather than the roll $(\phi)$ or pitch $(\theta)$. Nevertheless, only the latter two are corrected in our proposed method.

The unexpected results allows us to understand the limitations of our proposed method. In the current implementation, only the incoherences in the roll $(\phi)$ and pitch $(\theta)$ of the thigh can be observed. If the reason for incoherent motion lies in the heading, the corrections applied might not lead to an improved performance.

The integration of biomechanical constraints in the INS comes at a cost of a greater complexity. An additional IMU on the foot is required to detect the stance phase.

The results of this work are supported by the prior work conducted by Ahmadi et al. [9]. The latter use also the concept of coherent and incoherent motions to improve the process of gait reconstruction.

In contrast the state of the art approaches, e.g. [5], our approach requires no external infrastructure. Also the use of landmarks, and therefore its detection and association, is not necessary. It would be possible, nevertheless, to integrate these other techniques with our proposed method.

We consider the practical implications of our work relevant for the development of INSs. Namely, that it is possible to use biomechanical constraints to improve the performance of INSs. These biomechanical constraints are independent of external infrastructure or the detection of environmental elements like landmarks.

\section{CONCLUSION}

The goal of this work was to analyze whether human biomechanics can improve the position estimation of an INS. For that purpose, we analyzed a possible integration of biomechanical constraints in an inertial navigation system.

The conclusion of our work is that the errors in the orientation estimation of an INS translate into incoherent motions. By correcting the incoherent motions, the performance of an INS improves. The advantage of our approach is that it is based on the limits of human phisiology, and therefore it is applicable to all users.

\section{REFERENCES}

[1] Statistisches Bundesamt. (2016) Unfallentwicklung auf deutschen strassen 2015. [Online]. Available: https://www.destatis.de/DE/PresseService/Presse/Pressekonferenzen/ 2016/Unfallentwicklung_2015/unfallentwicklung_uebersicht.html

[2] What is a wearable device? [Online]. Available: http: //www.wearabledevices.com/what-is-a-wearable-device/

[3] E. Munoz Diaz, F. De Ponte Müller, and J. J. García Domínguez, "Use of the magnetic field for improving gyroscopes' biases estimation," Sensors, vol. 17, no. 4, p. 832, 42017.

[4] D. Pham and Y. Suh, "Pedestrian navigation using foot-mounted inertial sensor and LIDAR," Sensors, vol. 16, no. 1, p. 120, January 2016.

[5] Z. Chen, H. Zou, H. Jiang, Q. Zhu, Y. C. Soh, and L. Xie, "Fusion of WiFi, smartphone sensors and landmarks using the Kalman filter for indoor localization," Sensors, vol. 15, no. 1, pp. 715-732, 2015.

[6] E. Munoz Diaz, M. Caamano, and F. Sánchez, "Landmark-based drift compensation algorithm for inertial pedestrian navigation," Sensors, vol. 17, no. 7, p. 1555, Jul. 2017.

[7] I. Skog, J. O. Nilsson, P. Hndel, and A. Nehorai, "Inertial sensor arrays, maximum likelihood, and Cramer-Rao bound," IEEE Transactions on Signal Processing, vol. 64, no. 16, pp. 4218-4227, Aug. 2016.

[8] I. Skog, J. O. Nilsson, D. Zachariah, and P. Hndel, "Fusing the information from two navigation systems using an upper bound on their maximum spatial separation," in 2012 International Conference on Indoor Positioning and Indoor Navigation (IPIN), Nov. 2012, pp. $1-5$.

[9] A. Ahmadi, F. Destelle, L. Unzueta, D. S. Monaghan, M. T. Linaza, K. Moran, and N. E. O. Connor, "3D human gait reconstruction and monitoring using body-worn inertial sensors and kinematic modeling," IEEE Sensors Journal, vol. 16, no. 24, pp. 8823-8831, Dec. 2016.

[10] P. Corke, "A robotics toolbox for MATLAB," IEEE Robotics \& Automation Magazine, vol. 3, no. 1, pp. 24-32, 31996.

[11] I. A. K. MD, Physiology of the Joints: Volume 2 Lower Limb. Churchill Livingstone, 2010.

[12] E. Munoz Diaz, "Inertial pocket navigation system: Unaided 3D positioning," Sensors (Switzerland), vol. 15, no. 4, pp. 9156-9178, 2015.

[13] D. Bousdar Ahmed, E. Munoz Diaz, and S. Kaiser, "Performance comparison of foot- and pocket-mounted inertial navigation systems," in International Conference on Indoor Positioning and Indoor Navigation (IPIN). IEEE, 102016.

[14] J. W. David Titterton, Strapdown Inertial Navigation Technology. Institution of Engineering and Technology, 2004. 